\title{
A Jordan Surface Theorem for Three-Dimensional Digital Spaces
}

\author{
Ralph Kopperman, ${ }^{1}$ Paul R. Meyer, ${ }^{2 *}$ and Richard G. Wilson ${ }^{2} \dagger$ \\ ${ }^{1}$ Department of Mathematics, City College, \\ C.U.N.Y., New York, NY 10031, USA \\ ${ }^{2}$ Department of Mathematics and Computer Science, \\ Lehman College, C.U.N.Y., Bronx, NY 10468, USA
}

\begin{abstract}
Many applications of digital image processing now deal with threedimensional images (the third dimension can be time or a spatial dimension). In this paper we develop a topological model for digital three space which can be useful in this context. In particular, we prove a digital, three-dimensional, analogue of the Jordan curve theorem. (The Jordan curve theorem states that a simple closed curve separates the real plane into two connected components.) Our theorem here is a digital topological formulation of the Jordan-Brouwer theorem about surfaces that separate three-dimensional space into two connected components.
\end{abstract}

Topological properties of two-dimensional images on cathode ray tubes have been utilized and studied for some years, but so far there seems to be no consensus about how to extend these ideas to three-dimensional images (see, for example, [8] in reference to difficulties with shrinking algorithms).

A natural way to begin such a (two-dimensional) theory is to give a definition of connectedness for subsets of a digital plane which allows us to prove a Jordan curve theorem. (The Jordan curve theorem states that a simple closed curve separates a plane into two connected subsets, the inside and the outside.) The generally accepted approach to this has been a graph-theoretical Jordan curve theorem, due originally to Rosenfeld, which is not based on a topology and in fact requires two different definitions of connectedness, one for the curve (8-connectedness) and one for its complement (4-connectedness); see, for example, [13] or [14].

* BITNET address: PRMLC@CUNYVM.CUNY.EDU.

† Present address: Departamento de Matemáticas, Universidad Autónoma Metropolitana, Unidad Iztapalapa, México 13, D.F. 
This graph-theoretical approach has been refined and extended to threedimensional images; see [10], [11], [12], [6], and [7]. In [6] Kong and Roscoe prove a three-dimensional Jordan surface theorem which does not include the graph associated with our topological construction here (because 10-adjacency arises in our theory but not in theirs; see Fig. 4). In [7] Kong and Roscoe study a very general class of graph-theoretical settings for three-dimensional digital pictures (called "normal digital pictures"); this class does include the graph from our construction here, but they do not consider the Jordan surface theorem there. See also [9], where an approach similar to ours was proposed without proof.

In [5] we introduced a purely topological context for a digital plane and proved a Jordan curve theorem. ([5] was based on earlier work by Khalimsky; see [2]-[4].) This paper extends this approach to three dimensions by proving an analogous theorem for Jordan surfaces. By placing two- and three-dimensional computer graphics formally within point set topology, we get natural analogues of the usual topologies for two- and three-dimensional Euclidean space. The crucial step is to utilize a natural connected topology on a finite, totally ordered set; the topologies on the two- and three-dimensional digital spaces are then just the associated product topologies. Furthermore, this permits us to define path, arc, and curve using continuous functions on such a parameter interval.

In order to make this paper self-contained, we begin by summarizing the necessary background from [5]. We define a topology $\sigma$ on the set of integers $Z$ by specifying the minimal open (topological) neighborhoods at each point (see Fig. 1). There are two types of points, and they alternate. If $p$ is of one type, its minimal neighborhood is $N(p)=\{p-1, p, p+1\}$. Similarly, $N(p+2)=$ $\{p+1, p+2, p+3\}$. The intermediate point, $p+1$, must then have $N(p+1)=$ $\{p+1\}$ (as the intersection of two open sets). Thus $\{p-1\}$ and $\{p+1\}$ are open, while $\{p\}$ and $\{p+2\}$ are closed (their complements are open). Thus the points alternate between being open and closed. A COTS (connected ordered topological space) is an interval $C$ of $(Z, \sigma)$ with the inherited topology. (A more detailed discussion of COTS in a more general context is given in [5], but that given here is sufficient for the purposes of this paper.) If $C$ is finite, then the first and last points of $C$ are called its endpoints.

Now assume that $X_{1}, X_{2}, X_{3}$ are finite COTS and $X=X_{1} \times X_{2} \times X_{3}$ with the product topology $\tau$. Since this space, the digital counterpart of three-dimensional real space, is the setting for our main theorem, let us now sketch some of its properties, using three diagrams for illustration. All three of these diagrams deal

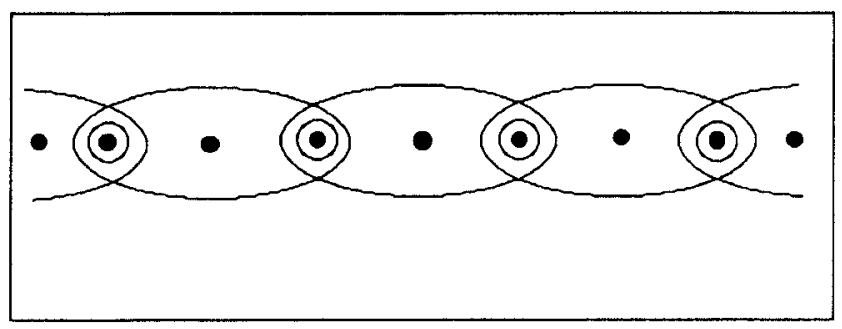

Fig. 1. A portion of a finite COTS showing the minimal neighborhoods of each point. 


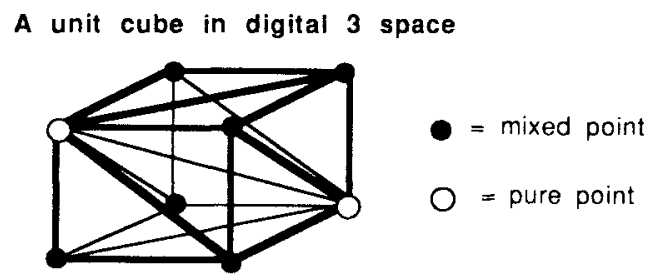

Fig. 2. A unit cube in $X$ showing which pairs of points are connected. Note that there is exactly one diagonal in each face and one internal diagonal which connects the two pure points.

with aspects of the question of which pairs of points are topologically connected. In the diagrams a line segment between two points indicates that they are topologically connected; this is purely schematic of course, since the digital space consists only of the points. (A more formal treatment, with detailed proofs, is given in [5].) Before giving the diagrams, we need some more definitions.

Definition. A dyad in $X$ is a connected two-point set. Similarly, a triad (resp. tetrad) is a mutually connected three- (four-) point set.

Definition. The adjacency set of a point $p \in X$ (written $\mathscr{A}(p))$ is the set of points, each of which forms a dyad with $p$. That is to say, $\mathscr{A}(p)=\{x \in X:\{p, x\}$ is connected and $x \neq p\}$.

It turns out that, in terms of adjacency sets, there are just two classes of points, which we now define. It is natural to call a point in $X$ open (resp., closed) if each of its coordinates is open (closed). We shall see that open and closed points behave similarly. A point in $X$ which is either open or closed is called pure; a point which is not pure is mixed.

We illustrate the topology of $X$ by showing the connectedness relationships in a unit cube of $X$ (Fig. 2); with an appropriate rotation and/or reflection, any unit cube in $X$ can be reduced to this configuration.

The adjacency set of a pure point is shown in Fig. 3, while the adjacency set of a mixed point is shown in Fig. 4.

Before proceeding to the main result of this paper we need definitions of digital analogues of arc, Jordan curve, and Jordan surface.

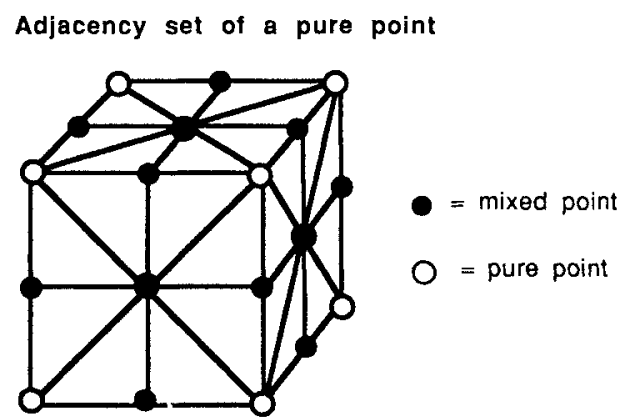

Fig. 3. This shows a portion of the adjacency set of a pure point; this pure point and the three other faces of the adjacency set are not shown here. 


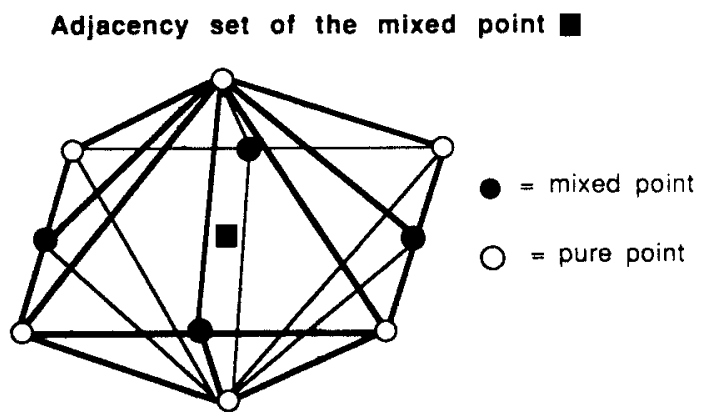

Fig. 4. This shows the complete adjacency set of a mixed point for the case in which the mixed point has the form open-open-closed or closed-closed-open (i.e., the $x$ and $y$ coordinates are of the same topological type). Recall that the center point does not belong to the adjacency set.

Definition. A digital arc is a homeomorphic image of a COTS. A digital Jordan curve is a connected subset $J$ with $|J| \geq 4$ such that $J-\{j\}$ is an arc for any $j \in J$. If no confusion can arise, we may omit the adjective digital.

If $J$ is a finite connected subset of a topological space with $|J| \geq 4$, then $J$ is a Jordan curve if and only if $|\mathscr{A}(j) \cap J|=2$ for each $j \in J$, in which case these two points are the endpoints of $J-\{j\}$ (proved in [5]). Furthermore, no proper subset of a digital Jordan curve can be a digital Jordan curve. (If $K$ is a proper nonvoid subset of a digital Jordan curve $J$, then $K$ has a point $p$ such that $|\mathscr{A}(p) \cap K|<2$; see 17(a) of [5].) Note that this also shows that no proper superset of a Jordan curve can be a Jordan curve.

Definition. The border of $X$ is the set of all points of $X$, at least one of whose coordinates is an endpoint of the corresponding $X_{i}(i=1,2,3)$.

Definition. A digital Jordan surface in $X$ is a $\tau$-connected subset $S$ of $X$ which does not meet the border of $X$ and such that for all $p \in S, \mathscr{A}(p) \cap S$ is a digital Jordan curve. The following lemma gives some properties which begin to justify calling such sets surfaces.

Lemma. If $S$ is a digital Jordan surface then:

(1) Each dyad in $S$ is contained in exactly two triads in $S$.

(2) $S$ contains no tetrads.

Proof. (1) Let $p \in S ; \mathscr{A}(p) \cap S$ is a digital Jordan curve, $J$ say. Observe that $\{p, q, r\}$ is a triad in $S$ if and only if $\{q, r\}$ is a dyad in $J$. However, $|\mathscr{A}(q) \cap J|=2$, and so it follows that for a given $p$ and $q$ there are exactly two such $r$.

(2) If $\{p, q, r, s\}$ is a tetrad in $S$, then $\{q, r, s\}$ is a triad in $S \cap \mathscr{A}(p)$ which is a contradiction since this latter set is a digital Jordan curve.

We are now in a position to prove the main theorem which is a digital analogue of the Jordan-Brouwer theorem for compact two-manifolds embedded in $R^{3}$ (proved for instance in Theorem 27.10 of [1]). 
Theorem. If $S$ is a digital Jordan surface in $X$, then $X \backslash S$ has exactly two $\tau$-components.

Proof. It is clear that the set $X$ is a subset of the integer lattice points of $R^{3}$. Let $\nu$ be the usual Euclidean topology for $R^{3}$. We define an operation ${ }^{\wedge}$ as follows: if $A$ is a subset of $X$, then $A^{\wedge}$ denotes the subset of $R^{3}$ formed by the union of all the isolated points and the (Euclidean) convex hulls of the dyads, triads, and tetrads contained in $A$. We now show that $S^{\wedge}$ is a compact two-manifold in $R^{3}$. To verify this it suffices to show that each $p \in S^{\wedge}$ has a neighborhood in $R^{3}$ whose intersection with $S^{\wedge}$ is homeomorphic to a 2-disc. We consider three cases:

(1) If $p$ lies in the interior of some triangular face of $S^{\wedge}$, then there is clearly an $\varepsilon>0$ such that $S^{\wedge} \cap B_{\varepsilon}(p)$ (the $\varepsilon$-ball centered at $p$ ) is homeomorphic to a 2-disc. We omit the details.

(2) If $p$ lies on a segment of $S^{\wedge}$ but is not a vertex, then, by part (1) of the lemma, $p$ lies on the boundary of precisely two triangular faces of $S^{\wedge}$. Again we omit the details, but it is clear that there is an $\varepsilon$-ball centered at $p$ whose intersection with $S^{\wedge}$ is a 2 -disc.

(3) Suppose now that $p$ is a vertex of $S^{\wedge}$. By hypothesis $\mathscr{A}(p) \cap S$ is a digital Jordan curve. Let $D$ be a dyad with $p \in D$, say $D=\left\{p, q_{1}\right\}$. Again by part (1) of the lemma, $D$ is contained in two triads, so choose one of them, $\left\{p, q_{1}, q_{2}\right\}$ say. The dyad $\left\{p, q_{2}\right\}$ is contained in one other triad, $\left\{p, q_{2}, q_{3}\right\}$ say. In this way we determine a finite set of elements $q_{1}, q_{2}, \ldots, q_{n}$ in $\mathscr{A}(p) \cap S$; by the finiteness of $\mathscr{A}(p)$, eventually one of the elements will repeat. Let $q_{n}$ be the first that repeats; $q_{n}=q_{j}, j<n$. Then $j=1$, since otherwise $q_{j-1}, q_{j+1}, q_{n-1}$ are distinct elements of the Jordan curve $\mathscr{A}(p) \cap$ $S$, each of which is adjacent to $q_{n}$, a contradiction. The set of $q_{i}$ 's has the property that each member is in the adjacency set of two other members. Such a set cannot be a proper subset of a digital Jordan curve, and so the $q_{i}$ 's must exhaust the digital Jordan curve $\mathscr{A}(p) \cap S$. Now as before, it is clear that there is $\varepsilon>0$ such that $B_{\varepsilon}(p) \cap S^{\wedge}$ is a 2 -disc.

Thus we have shown that $S^{\wedge}$ is a 2 -manifold; being a finite union of triangles, it is compact. The Jordan-Brouwer theorem states that a compact 2-manifold separates $R^{3}$ into two $\nu$-components $I_{e}$ and $E_{e}$ (the inside and the outside of $\left.S^{\wedge}\right)$. Let $I=X \cap I_{e}$ and $E=X \cap E_{e}$. It remains to show that $I$ and $E$ are the nonempty $\tau$-components of $X \backslash S$, that is to say, $I$ and $E$ are $\tau$-separated, $\tau$-connected, and nonempty.

(a) We first show that $I$ and $E$ are $\tau$-separated. To this end, assume to the contrary. It follows (see Theorem 13(c) of [5]) that there are points $p \in I$ and $q \in E$ such that $\{p, q\}$ is $\tau$-connected. The segment $p q$ in $R^{3}$ has one endpoint $p$ in $I_{e}$ and the other endpoint $q$ in $E_{e}$, hence $p q$ must meet $S^{\wedge}$. Now segment $p q$ does not meet $S$, since $p$ and $q$ are not in $S$ and they are the only points on $p q$ with integer coordinates. So $p q$ meets $A^{\wedge}$ for some dyad or triad $A$ in $S$. It is clear that every dyad or triad in $X$ must be contained in some unit lattice cube like that pictured in Fig. 2. We see from that diagram that if a dyad $D$ has no points in common with a dyad 
or triad $W$, then $D^{\wedge}$ and $W^{\wedge}$ are disjoint. This contradicts the fact that the segment $p q=\{p, q\}^{\wedge}$ meets $A^{\wedge}$, and the contradiction proves (a).

(b) We now show that $I$ is $\tau$-connected and nonvoid (the proof for $E$ is similar). Since $I_{e}$ is connected and nonvoid it suffices to construct a continuous surjection $f:\left(I_{\epsilon}, \nu\right) \rightarrow(I, \tau)$. We construct $f$ as a composition $\pi \circ \rho$ of continuous functions, where $\rho:\left(I_{e}, \nu\right) \rightarrow\left(I^{\wedge}, \nu\right)$ and $\pi:\left(I^{\wedge}, \nu\right) \rightarrow$ $(I, \tau)$. (In fact $\pi$ is a quotient mapping and $\rho$ is a retraction, but we do not need these further properties.)

To define $\pi$, we first construct $\pi_{1}$, a continuous quotient mapping of the reals $R$ with the usual topology onto the integers $Z$ with a COTS topology. Since $X$ was identified as a set of integer lattice points in $R^{3}$, the desired $\pi$ is then obtained as the restriction to $I^{\wedge}$ of the product mapping $\pi_{2}=\pi_{1} \times \pi_{1} \times \pi_{1}$. Assuming for definiteness that $Z$ has the COTS topology in which the even integers are closed and the odd integers are open, we can for example define $\pi_{1}$ by $\pi_{1}(2 n)=2 n$ and $\pi_{1}(x)=2 n+1$ if $2 n<x<2 n+2$. If $T^{\wedge}$ is the tetrahedron arising from a $\tau$-tetrad $T$, it follows from the definition of $\pi_{1}$ that $\pi_{2}$ maps $T^{\wedge}$ onto its set of vertices $T$; similarly for dyads and triads. Hence $\pi$ maps $I^{\wedge}$ onto $I$, as required.

It remains to construct $\rho$. Since some points in $I$ are $\tau$-connected to points of $S$, we must retract $I_{e}$ onto $I^{\wedge}$ before taking the quotient. Thus we define $\rho$ to be the identity mapping on $I^{\wedge}$. $I_{e}-I^{\wedge}$ consists of all segments, triangles, and tetrahedra which are incomplete in the sense that they lack vertices, edges, and/or faces which lie in $S$. We will define $\rho$ on each such segment, triangle, or tetrahedron in a consistent manner, so that we can be sure that the definitions agree on the intersection of adjacent (closed in the subspace topology) pieces. The resulting function is then continuous. (If a function is defined on a finite union of closed subsets so that its restriction to each such subset is continuous, then the function itself is continuous [15, Theorem 7.6].)

Case 1. If a segment, triangle, or tetrahedron has just one vertex in $I^{\wedge}$, then map it onto that vertex.

Case 2.

(i) If a triangle has only two vertices in $I^{\wedge}$, then project from the missing vertex onto the edge determined by the other two.

(ii) If a tetrahedron $T^{\wedge}$ has only two vertices in $I^{\wedge}$, then each point of $T^{\wedge}-I^{\wedge}$, together with these two vertices, determines a plane whose intersection with $T^{\wedge}$ is a triangle; project in that triangle as in Case 2(i).

Case 3. If a tetrahedron has exactly three vertices in $I^{\wedge}$, then project from the missing vertex onto the triangle in $I^{\wedge}$.

We now show that the union of the segments, triangles, and tetrahedra considered in Cases 1-3 contains the whole of $I_{e}-I^{\wedge}$. If $p \in I_{e}$ is not a lattice point, then $p$ lies in the Euclidean interior of some segment (or triangle or tetrahedron) defined by a dyad (or a triad or a tetrad) of $X$. If none of the vertices of this segment (respectively triangle, tetrahedron) lie in $I_{e}$, then they must lie in $S$ (since $I_{e}$ and $E_{e}$ are separated by $S^{\wedge}$, they do not lie in $E_{e}$ ). But since $S^{\wedge}$ 
contains the convex hull of every dyad, triad, and tetrad in $S$ it follows that $p \in S^{\wedge}$ which is a contradiction. This completes the proof that $\rho$ is well defined and also shows that $I$ is nonvoid, thereby completing the proof of the theorem.

We have thus completed the proof of the digital analogue of the threedimensional Jordan-Brouwer theorem. While we make no attempt at a proof here, a similar method can be used to prove a digital analogue of the (two-dimensional) Jordan curve theorem (see [5]).

\section{Acknowledgments}

We thank Efim Khalimsky, Yung Kong, Erwin Kronheimer and Connor Lazarov for their helpful comments.

\section{References}

1. Greenberg, M., Lectures on Algebraic Topology, Benjamin, New York, 1967.

2. Khalimsky, E. D. (Halimskii, E.), On topologies of generalized segments, Soviet Math. Dokl. 10 (1969), 1508-1511.

3. Khalimsky, E. D., Applications of connected ordered topological spaces in topology. Conference of Mathematics Departments of Povolsia, 1970.

4. Khalimsky, E. D., Ordered Topological Spaces, Naukova Dumka, Kiev, 1977.

5. Khalimsky, E. D., Kopperman, R., and Meyer, P. R., Computer graphics and connected topologies on finite ordered sets, Topology Appl. (in press).

6. Kong, T. Y., and Roscoe, A. W., Continuous analogs of axiomatized digital surfaces, Comput. Vision Graphics Image Process. 29 (1985), 60-86.

7. Kong, T. Y., and Roscoe, A. W., A theory of binary digital pictures, Comput. Vision Graphics Image Process. 32 (1985), 221-243.

8. Kong, T. Y., and Rosenfeld, A., Digital topology: introduction and survey, Comput. Vision Graphics Image Process. (in press).

9. Kovalevsky, V. A., On the topology of discrete spaces, Studientexte, Digitale Bildverarbeitung, Heft 93/86, Technische Universitat Dresden, 1986.

10. Morgenthaler, D. G., and Rosenfeld, A., Surfaces in three-dimensional digital images, Inform. and Control 51 (1981), 227-247. MR 84j:68062.

11. Reed, G. M., On the characterization of simple closed surfaces in three-dimensional digital images, Comput. Vision Graphics Image Process. 25 (1984), 226-235.

12. Reed, G. M., and Rosenfeld, A., Recognition of surfaces in three-dimensional digital images, Informat. and Control 53 (1982), 108-120.

13. Rosenfeld, A., Digital topology, Amer. Math. Monthly 86 (1979), 621-630.

14. Rosenfeld, A., Picture Languages, Academic Press, New York, 1979.

15. Willard, S., General Topology, Academic Press, New York, 1970.

Received August 3, 1988, and in revised form January 5, 1989. 